\title{
Investigation on Dabigatran Etexilate and Worsening of Renal Function in Patients with Atrial fibrillation: The IDEA Study
}

\author{
Mauro Molteni ${ }^{1} \cdot$ Matteo Crippa $^{2} \cdot$ Annalisa Orenti $^{3} \cdot$ Hernan Polo Friz $^{2,5} \cdot$ Anna \\ Menghini $^{2} \cdot$ Pierluigi Tramacere $^{4}$ - Giuseppe Marano $^{3} \cdot$ Claudio Cimminiello $^{5} \cdot$ Patrizia \\ Boracchi $^{3}$
}

1 UOC Cure Subacute, IRCCS, Istituti Clinici Scientifici Maugeri, via Camaldoli 64, 20138 Milan, Italy

2 Internal Medicine, Vimercate Hospital, A.S.S.T. di Vimercate, via Santi Cosma e Damiano 10, 20871 Vimercate,

3 Department of Clinical Sciences and Community Health, Laboratory of Medical Statistics, Epidemiology and Biometry G. A. Maccacaro, University of Milan, via Vanzetti 5, 20133 Milan, Italy

4 Clinical Pathology, Vimercate Hospital, A.S.S.T. di Maugeri, via Camaldoli 64, 20138 Milan, Italy Vimercate, via Santi Cosma e Damiano 10, 20871 Vimercate, MB, Italy

5 Studies and Research Office of Italian Society of Angiology MB, Italy and Vascular Pathology (SIAPAV), via Gorizia 22, 20144 Milan, Italy

Corresponding Author:

Mauro Molteni

UOC Cure Subacute, IRCCS, Istituti Clinici Scientifici Maugeri, via Camaldoli 64, 20138 Milan, Italy

Mail: mauro.molteni@icsmaugeri.it 


\begin{abstract}
Background and Objectives Warfarin-related nephropathy is an unexplained acute kidney injury, and may occur in patients with supratherapeutic INR, in the absence of overt bleeding. Similar findings have been observed in rats treated with dabigatran etexilate. We conducted a prospective study in dabigatran etexilate-treated patients to assess the incidence of dabigatranrelated nephropathy and to investigate the possible correlation between dabigatran plasma concentration (DPC) and worsening renal function.
\end{abstract}

Method One hundred and seven patients treated long term with dabigatran etexilate for nonvalvular atrial fibrillation (NVAF) were followed up for 90 days. DPC, serum creatinine (SCr) and serum cystatin $\mathrm{C}$ were prospectively measured. Ninety five patients had complete follow-up data and were evaluable for primary endpoint.

Results Eleven patients had supratherapeutic DPC, defined as DPC higher than $200 \mathrm{ng} / \mathrm{ml}$ at study enrolment, but at the end of follow-up no patient showed a persistent increase in SCr. No patients experienced acute kidney injury.

Conclusions Our study shows that no persistent renal detrimental effect is associated with dabigatran treatment. An increase in SCr during dabigatran treatment is reversible and it seems to be unrelated to dabigatran itself. 


\section{Introduction}

Anticoagulant therapy with vitamin $\mathrm{K}$ antagonists (VKAs) and, more recently, with direct oral anticoagulants (DOACs) is the mainstay of stroke prevention in non-valvular atrial fibrillation (NVAF) [1]. Despite being highly effective with a stroke rate reduction of nearly $62 \%$ compared with placebo [2], anticoagulant agents are associated with major bleeding that can eventually be lethal [3]. Non-haemorrhagic adverse events with anticoagulation may also occur, although quite rarely [4-8].

Recently, a new complication of VKAs has been described, i.e. warfarin-related nephropathy (WRN), also

known as warfarin-related renal impairment. It consists of an unexplained acute kidney injury, defined as a serum creatinine ( $\mathrm{SCr}$ ) increase greater than $0.3 \mathrm{mg} / \mathrm{dl}$ within 1 week of a supratherapeutic INR (international normalised ratio), without overt bleeding [9-14]. WRN seems to reduce 1-year survival and to accelerate renal dysfunction, especially but not only in patients with pre-existing chronic kidney disease (CKD) $[10,12,13]$. As it has been proposed that WRN could be due to glomerular micro-bleeds $[15,16]$, the same complication could also occur with DOACs. Indeed, similar findings have been observed in rats treated with dabigatran etexilate [17]. Alternatively, more recently, renal tubule occlusion by red blood cells has been suggested as a possible pathogenic mechanism [18].

Based on such evidence, we conducted a prospective study in patients treated with dabigatran for NVAF to assess the incidence of anticoagulant-related nephropathy and to investigate whether dabigatran plasma concentration (DPC) was related to the worsening of renal function.

\section{Patients and Methods}

\section{Study Overview}

This was an observational prospective single-centre study. Our local Ethics Committee approved the study. All patients provided written informed consent. The primary analyses of the data were performed in the Laboratory of Medical Statistics, at the Department of Clinical Sciences and Community Health, University of Milan, Milan, Italy.

\section{Study Design}


Patients on treatment with dabigatran etexilate with NVAF were prospectively followed at four time-points: T0

(enrolment time), T1 (7 days after enrolment), T2 and T3 (60 and 90 days after enrolment, respectively). Time-point spacing was based on previous studies focusing on WRN. It is known that patients developing WRN show a peak in SCr during the first week, then SCr remains elevated for about 3 months, slowly recovering but never returning to baseline levels. Blood samples were harvested to determine DPC, measured as diluted thrombin time (dTT, HEMOCLOT $\left.{ }^{\circledR}\right)[19,20]$, at T0 and T1; SCr and serum cystatin $\mathrm{C}(\mathrm{CysC})$ were measured at all four time points. Figure 1 summarizes the study design: TSTART is the time point when dabigatran etexilate therapy was started and refers to $\mathrm{SCr}$ available at that moment.

\section{Study Outcomes}

The primary objective of this study was to estimate the incidence of (unexplained) renal injury at 90 days in patients with a DPC higher than the therapeutic range at enrolment. DPC, measured as dTT, was defined as supra-therapeutic, if higher than $200 \mathrm{ng} / \mathrm{ml}$. This is in accordance with the practical guide on management of DOACs [21] proposed by the European Heart Rhythm Association (EHRA). Unexplained acute renal injury was defined, according to the WRN definition, as an increase in $\mathrm{SCr}$ of greater than $0.3 \mathrm{mg} / \mathrm{dl}$ from enrolment to 90 days, without clinical evidence of haemorrhage.

The secondary objective of this study was to evaluate the incidence of renal damage at each time point during follow-up and to assess the persistence or regression of kidney function impairment at the different time points of the study.

\section{Study Population}

All the patients followed for NVAF at ASST Vimercate, Department of Internal Medicine, (Vimercate, MB, Italy) were assessed for eligibility. Inclusion criteria were age $>18$ years, confirmation of non-valvular cardiomyopathy [22] by colour Doppler echocardiography, ongoing treatment with dabigatran etexilate, and ability to sign the informed consent form. Patients were excluded in case of inability to follow the study protocol, altered thyroid function during the last 6 months, severe renal failure $(<30 \mathrm{ml} / \mathrm{min})$, and concomitant corticosteroid therapy. The following information was collected at baseline (T0): age, sex, body mass index (BMI), and dabigatran etexilate dosage (e.g. $110 \mathrm{mg}$ twice daily (BID) or $150 \mathrm{mg}$ BID), and 
dabigatran etexilate therapy duration. CHA2DS2-VASc [23] and HAS-BLED scores [24] were calculated for each patient. A relevant medical history was collected, and concurrent medications were recorded.

Follow-up was planned 1 week after enrolment (T1), at 2 months (T2) and at 3 months ( T3): relevant medical information, such as hospitalizations, cardiovascular events and variation in concomitant medications were recorded.

\section{Sample Material and Laboratory Methods}

At all time-points, blood samples were collected to determine $\mathrm{SCr}$ and $\mathrm{CysC}$. Furthermore, at study enrolment and 1 week later (respectively, T0 and T1) blood samples were collected to determine DPC. Blood samples were harvested at trough concentration of dabigatran (e.g. just before dabigatran etexilate intake), via a standard procedure (cubital/cephalic vein puncture with butterfly/Vacutainer ${ }^{\circledR}$ ), and then immediately centrifuged (3500 rpm for $12 \mathrm{~min}$, at room temperature). The tubes were then stored at $-80^{\circ} \mathrm{C}$ until analysis. DPC measured as dTT and expressed as $\mathrm{ng} / \mathrm{ml}$ was determined on defrosted platelet-free plasma, derived from citrated blood samples, with the HEMOCLOT $₫$ assay [19,20], on a BCS-XP analyser (Siemens Healthcare Diagnostics, Marburg, Germany). SCr and CysC samples were collected in serum-separating tubes and were submitted to the same centrifugation and storage process. Serum concentration was measured by Roche-Hitachi Cobas 6000, Analytical system.

\section{Statistical Analysis}

Demographic and clinical characteristics of patients were summarized by absolute numbers and percentages for categorical variables and by median, first and third quartiles for quantitative variables. For descriptive purposes, the variable distribution was reported firstly within two groups of subjects according to the dabigatran etexilate dosage (110 mg BID or $150 \mathrm{mg}$ BID) and secondly within two groups of subjects according to DPC at T0 $(<200 \mathrm{ng} / \mathrm{ml}, \geq 200 \mathrm{ng} / \mathrm{ml})$. The variable distribution between the two groups of patients mentioned above was compared using Fisher's exact test for categorical variables and the non-parametric Wilcoxon rank sum tests for continuous variables.

To evaluate the relationship between DPC at T0 (response) and age (explanatory variable) a linear regression model was fitted.

To evaluate the incidence of renal damage at T3 (primary endpoint) the inference was based on 
95\% confidence intervals (CIs). Fisher's exact method was applied [25].

To evaluate renal damage at each time-point during the follow-up (secondary endpoint) the proportion of renal damage was computed with the pertinent $95 \%$ CI obtained

with exact method. The incidence of renal damage, according to age, sex, BMI and daily dabigatran etexilate dosage assumption, was estimated by a generalized linear regression model with binomial error on all patients with complete information on the occurrence of renal damage from $\mathrm{T} 0$ to $\mathrm{T} 3$.

Furthermore, evaluation of the persistence or regression of renal impairment during 90 days of follow-up was performed through a mixed-model regression analysis [26] on all patients with complete information from $\mathrm{T} 0$ to $\mathrm{T} 3$. At the beginning of the study, it was not possible to compute sample size because no information was available on the expected number of renal injuries in patients with supra-therapeutic DPC. For practical considerations, the study was based on a 1-year recruitment (about 100 patients expected).

At the beginning of the study, it was not possible to compute sample size because no information was available on the expected number of renal injuries in patients with supra-therapeutic DPC. For practical considerations, the study was based on a 1-year recruitment (about 100 patients expected).

\section{Results}

From 1July to 23 October 2015 we recruited 107 patients on dabigatran etexilate for NVAF. Sixty-one patients ( $57 \%$ of the overall study population) were receiving dabigatran etexilate 110 $\mathrm{mg}$ BID and the remaining 43\% dabigatran etexilate $150 \mathrm{mg}$ BID. The median age was 79 years and the median CHA2DS2-VASc score was five, while the median HAS-BLED score was three.

Patient characteristics are described in Table 1, accordingto dabigatran etexilate dosage. The patients treated with $110 \mathrm{mg}$ BID were older than those treated with $150 \mathrm{mg}$ BID (median age 83 years vs. 73 years, $\mathrm{p}<0.001)$ and were more frequently women $(59 \%$ vs. $37 \%, \mathrm{p}=0.032)$. Patients treated with the two dosages had a similar DPC, both at T0 and T1: median DPC at T0 was $106.3 \mathrm{ng} / \mathrm{ml}$ in the dabigatran etexilate $110 \mathrm{mg}$ group and $99 \mathrm{ng} / \mathrm{ml}$ in dabigatran etexilate $150 \mathrm{mg}(\mathrm{p}=0.389)$ and median DPC at T1 was $128.9 \mathrm{ng} / \mathrm{ml}$ in dabigatran etexilate $110 \mathrm{mg}$ group versus $99.2 \mathrm{ng} / \mathrm{ml}$ in dabigatran etexilate $150 \mathrm{mg}$ group $(\mathrm{p}$ 
$=0.072$ ).

Table 2 shows the demographic and clinical characteristics for patients with therapeutic DPC and supratherapeutic DPC. We observed that CHA2DS2-VASc distributions were significantly different according to DPC at T0 $(p=0.015)$.

The median CHA2DS2-VASc in patients with therapeutic DPC was four, whereas the median CHA2DS2-VASc in patients with supratherapeutic DPC was five. When considering $\mathrm{SCr}$ at Tstart, no statistically significant difference was observed between patients with lownormal DPC $(<200 \mathrm{ng} / \mathrm{ml})$, and patients with higher DPC: median SCr was $0.92 \mathrm{mg} / \mathrm{dl}$ in patients with therapeutic DPC and $0.98 \mathrm{mg} / \mathrm{dl}$ in patients with supratherapeutic DPC at T0 $(p=0.486)$.

With regard to age distribution according to DPC at T0, median age was higher in patients with supra-therapeutic DPC, even though the difference was not statistically significant. Moreover, when DPC was considered as a continuous variable, a statistically significant association between age and DPC was obtained: increasing age was associated with increasing DPC, as evaluated by linear regression model results. At T0, mean DPC increased by $2.672 \mathrm{ng} / \mathrm{ml}(95 \%$ CI 1.007-4.337) each year of increase in age.

Table 3 describes concomitant drug usage in the study population and according to DPC at T0. No influence of the different concomitant drugs was observed on DPC.

Due to technical reasons (e.g. tube breakage during defrosting procedures), DPC data were missing in two patients at T0. From T0 to T1 one patient, from T1 to T2 five patients and from $\mathrm{T} 2$ to $\mathrm{T} 3$ four patients were lost to follow-up. For these ten patients the exact withdrawn time was unknown. Of the remaining 95 patients with complete follow-up information, 11 had supratherapeutic DPC at T0. Thus, the primary endpoint was assessed on 11 patients. In this subset, the incidence of renal injury at T3 was zero (95\% CI 0-0.285). On the other hand, in the 84 patients with therapeutic DPC, only two patients showed a SCr increase higher than $0.3 \mathrm{mg} / \mathrm{dl}$ at T3 for a total incidence of 0.024 (95\% CI $0.003-0.083)$. There was no statistically significant difference in renal injury at the end of follow-up between patients with therapeutic or supra-therapeutic DPC at T0. The difference in incidence values was 0.024 (95\% CI -0.234 to 0.083 ). No patients needed a dabigatran dosage modification according to renal function. No dosage was modified throughout the study according supratherapeutic 
concentration of the drug. Furthermore, no patients experienced acute kidney injury.

If we consider all time points, 15 patients had supratherapeutic DPT at T0; one patient was lost to follow-up just after T0. From T0 to T1, the information was available for 14 patients, and among these, a SCr increase was present in one patient (proportion of renal damage: $0.714,95 \%$ CI $0.002-0.339$ ). From T1 to T2, the information was available of renal damage: $0.167,95 \%$ CI 0.0209-0.484). From T2 to T3, the information was available in 11 patients and one of them had renal damage (proportion of renal damage: $0.091,95 \%$ CI 0.002-0.413). If we consider all time points, 90 patients had therapeutic DPC at T0. Renal damage was observed in six out of 90 patients, from T0 to T1 (proportion of renal damage: $0.067,95 \% \mathrm{CI} 0.025-0.139$ ). SCr increase occurred in four patients out of 87 from T1 to T2 (proportion of renal damage: 0.046, 95\% CI 0.013-0.114), and in five patients out of 84 from T2 to T3 (proportion of renal damage: $0.060,95 \%$ CI $0.020-0.133)$.

Due to the small number of patients and renal injuries, we could not perform a multivariate analysis to correlate renal injury with the different variables. We therefore performed univariate analysis and found that no covariates (age, sex, BMI and dabigatran etexilate dosage) were significantly associated with renal damage. To evaluate the persistence of renal damage, in the entire sample of 95 patients with complete follow-up information, we observed that among the patients who developed SCr damage at T1, 59.4\% (95\% CI 8-100, p $=0.353)$ of them showed persistence of $\mathrm{SCr}$ damage at $\mathrm{T} 2$ too. No patients, however, showed persistence of $\mathrm{SCr}$ damage at T3. Therefore, we can affirm that in our study population, the SCr increase observed in dabigatran etexilate-treated patients was reversible in all cases.

\section{Discussion}

In recent years, WRN has been described $[9,10]$. Although it is more frequent in patients with $\mathrm{CKD}$, recent studies suggest that it can also occur in any VKA-experienced patients, and it is associated with age, diabetes mellitus, hypertension and cardiovascular disease [10]. Evidence suggests that WRN may influence mortality, and is associated with progressive loss of renal function [11-13]. Regardless of warfarin use, a renal injury could theoretically occur in any patient treated with anticoagulant therapy and no prospective data are available on DOACs. A few case reports have been published describing a possible kidney injury related to dabigatran 
etexilate $[17,18]$ and apixaban [27]. The present study is the first observational prospective investigating a relationship between DPC and renal injury, in patients affected by AF and treated with dabigatran etexilate. Retrospective analysis by Böhm, pertaining to RE-LY trial [28], shows that compared to warfarin-treated patients, dabigatran etexilate treatment was associated with a lower incidence of renal function decline. Renal impairment was amplified in diabetic patients and in experienced VKA users. The same study showed that patients who were in the therapeutic range for a short time exhibited the most rapid decline in GFR [29]. In a Taiwanese nationwide retrospective cohort study in Asian patients with NVAF, dabigatran etexilate was associated with a lower risk of acute kidney injury compared to warfarin, for patients with CKD and those without CKD [30]. Furthermore, a retrospective study on 9769 patients with NVAF showed that dabigatran etexilate, rivaroxaban and apixaban were associated with a reduced risk of $\geq 30 \%$ decline in eGFR, and doubling of SCr and AKI compared with warfarin [31]. In the 107 patients we enrolled, 57\% were treated with dabigatran etexilate 110 BID and, as expected, they were older than those treated with the highest dose. There was no difference in Tstart renal function between dabigatran etexilate $110 \mathrm{mg}$ and dabigatran etexilate $150 \mathrm{mg}$ subjects, and those treated with either of the two doses had comparable DPCs but there was a non-significant trend to a higher DPC in patients taking the lower dosage. Linear regression analysis showed that DPC increases by a factor of $2.6-3.3 \mathrm{ng} / \mathrm{ml}$ with each increase in year of age. This is likely due to a progressive impairment of drug metabolism, in association to patient ageing. Eikelboom et al. in a pre-specified subanalysis of the RE-LY trial showed that there is a significant treatment-by-age interaction. The interaction with age is evident for extracranial bleeding, but not for $\mathrm{ICH}$, with the risk of the latter being consistently reduced with both dosages of dabigatran etexilate, compared with warfarin, irrespective of age [32]. Our findings confirm Owada's results showing a high DPC in high-risk patients (fragile patients with a high CHA2DS2- VASc score) [33].

The primary outcome of our study was the incidence of renal injury at 3 months in patients with T0 supratherapeutic DPC (per EHRA definition) [21]. In the 11 patients withthe highest DPC at T0 (e.g. DPC $\geq 200 \mathrm{ng} / \mathrm{ml}$ ), no patient showed a SCr increase higher than $0.3 \mathrm{mg} / \mathrm{dl}$ at 3 months. As a secondary outcome, we considered all patients with therapeutic or supratherapeutic DPCs and we observed persistence of renal damage at different timepoints. Among the seven patients who developed renal damage between $\mathrm{T} 0$ and $\mathrm{T} 1,59.4 \%$ showed persistence of 
SCr damage at T2 and none of them showed persistence of renal injury at T3.

Therefore, it appears that in our study population, the SCr increase observed in dabigatran etexilate-treated patients was reversible in all cases. These data, so far, do not completely clarify whether a variation in DPC induces renal injury or vice versa. Nevertheless, as far as the results of the present study are concerned, we can state that a high DPC itself does not seem to induce an SCr increase. After renal excretion, DPC is higher in patients with a higher $\mathrm{SCr}$ and CysC. Dabigatran etexilate does not have a deleterious effect on renal function but renal function itself influences DPC. Patients with different DPCs (e.g. supratherapeutic vs. therapeutic DPC, regardless of the definition) had similar median SCr levels, and displayed a similar rate of $\mathrm{SCr}$ increase.

This study has several limitations, which are worth considering. First, our results are derived from a relatively small number of patients, and therefore the results cannot be extrapolated to another population. Furthermore, although this study was designed to identify patients with high DPC at trough during dabigatran etexilate treatment, the small sample size does not yet allow us to suggest a possible DPC cut-off for identifying patients with a higher risk of renal injury during DE treatment. Assuming an incidence of warfarin-related nephropathy of $10 \%$ in patients with no CKD, we would have expected at least one or two occurrences of dabigatran-related nephropathy. Since we found an incidence of $11.6 \%$ of patients with supra-therapeutic DPC in our population, we would probably need at least twice or three times as many patients to properly investigate this. Most recruited patients were prescribed dabigatran etexilate during hospitalization, mainly because of acute heart failure. This explains the high-risk profile, and could explain the initial renal impairment, which later, over time, improved and stabilized.For this reason, our sample reflects a highrisk population that would infrequently be enrolled in major phase III trials. Though dosage choices were made according to international recommendations [21] and to the Summary of Product Characteristics [34], we cannot exclude a physician influence on dosage prescription. We did not set any exclusion criteria on previous dabigatran etexilate therapy duration, but we did not find any influence on DPC per dabigatran etexilate treatment duration. The time point allocations can be questioned, but we decided to repeat DPC, SCr, CysC and eGFR after 1 week because in WRN kidney damage peaks 1 week after supratherapeutic INR measurement. Furthermore, we decided to repeat the measurement of renal function 3 months later, because WRN persists to 90 days and we wanted to observe 
whether a high DPC had long-lasting consequences for kidney function. We arbitrarily set a second time point at 2 months. We deliberately did not perform urinalysis. WRN is defined by an increase in $\mathrm{SCr}$ and not by by haematuria; indeed haematuria is an exclusion criterion. Monitoring of microscopic haematuria could have been an exploratory endpoint, but we decided to focus on an SCr increase and kidney function. Lastly, although WRN can occur also in patients with normal renal function, it is more frequently encountered in patients with CKD. Our population had a median $\mathrm{SCr}$ of $0.93 \mathrm{mg} / \mathrm{dl}$ and a median eGFR of $63.01 \mathrm{ml} / \mathrm{min}$. A larger population study could help to define whether patients with moderate CKD would have a higher risk of renal function impairment as a result of dabigatran etexilate treatment. Patients with severe CKD cannot be treated with dabigatran [34]. Aside from the several limitations, this is the first study specifically designed to evaluate the presence and the incidence of a dabigatran-related renal impairment in humans. It is a prospective study and is the first study to evaluate renal impairment through more sensible and specific methods such as CysC and the 2012 CKD-EPI cystatin C-creatinine formula.

\section{Conclusion}

In conclusion, our study does not show any persistent detrimental effect of dabigatran etexilate itself on renal function. We did not find any connection between high DPC and renal injury. SCr increases during dabigatran etexilate treatment are reversible and are probably not related to the drug itself. However, since the renal events were few and all were reversible, this study does not provide much in answering how many patients would be needed in a future study to definitively exclude or confirm the existence of dabigatran-related nephropathy.

\section{Acknowledgements}

We are grateful to Dr. Maria Cristina Straface and Giuseppina Caiazzo for laboratory measurements, and to the nurses of the medical ward "Tulipano Rosso" of Vimercate Hospital, for harvesting blood samples. 


\section{References}

1. Hart RG, Benavente O, McBride R, Pearce LA. Antithrombotictherapy to prevent stroke in patients with atrial fibrillation: a metaanalysis. Ann Intern Med. 1999;131:492-501.

2. Ruff CT, Giugliano RP, Braunwald E, Hoffman EB, DeenadayaluN, Ezekowitz MD, Camm AJ, Weitz JI, Lewis BS, Parkhomenko A, Yamashita T, Antman EM. Comparison of the efficacy and safety of new oral anticoagulants with warfarin in patients with atrial fibrillation: a metaanalysis of randomized trials. Lancet. 2014;383:955-62.

3. Flaherty ML, Kissela B, Woo D, Kleindorfer D, Alwell K, SekarP, Moomaw CJ, Haverbusch M, Broderick JP. The increasingincidence of anticoagulant-associated intracerebral hemorrhage.Neurology. 2007;68:116-21.

4. Verhagen H. Local hemorrhage and necrosis of the skin and underlying tissues at starting therapy with dicumarol or dicumacyl.Acta Med Scand. 1954;148:453-67.

5. Raj K, Collins B, Rangarajan S. Purple toe syndrome following anticoagulant therapy. Br J Haematol. 2001;114:740.

6. Hauschka PV, Lian JB, Cole DEC, Gundberg CM. Osteocalcin and matrix Gla protein: vitamin K-dependent proteins in bone. Physiol Rev. 1989;69:990-1047.

7. Pettifor JM, Benson R. Congenital malformations associated with the administration of oral anticoagulants during pregnancy. J Pediat. 1975;86:459-62.

8. Danziger J. Vitamin K-dependent proteins, warfarin, and vascular calcification. Clin J Am Soc Nephrol. 2008;3:1504-10.

9. Brodsky SV, Satoskar A, Chen J, Nadasdy G, Eagen JW, Hamirani M, Hebert L, Calomeni E, Nadasdy T. Acute kidney injury during warfarin therapy associated with obstructive tubular red blood cell casts: a report of 9 cases. Am J Kidney Dis. 2009;54:1121-6.

10. Brodsky SV, Nadasdy T, Rovin BH, Satoskar AA, Nadasdy GM, Wu HM, Bhatt UY, Hebert LA. Warfarin-related nephropathy occurs in patients with and without chronic kidney disease and is associated with an increased mortality rate. Kidney Int. 2011;80:181-9.

11. Moreno JA, Martín-Cleary C, Gutiérrez E, Toldos O, Blanco- Colio LM, Praga M, Ortiz A, Egido J. AKI associated with macroscopic glomerular hematuria: clinical and pathophysiologic consequences. Clin J Am Soc Nephrol. 2012;7:175-84.

12. An JN, Ahn SY, Yoon CH, Youn TJ, Han MK, Kim S, Chin HJ, Na KY, Chae DW. The occurrence of warfarin-related nephropathy and effects on renal and patient outcomes in korean 
patients. PLoS One. 2013;8:e57661. https ://doi.org/10.1371/journ al.pone.00576 61.

13. Lim AK, Campbell DA. Haematuria and acute kidney injury in

elderly patients admitted to hospital with supratherapeutic warfarin

anticoagulation. Int Urol Nephrol. 2013;45:561-70.

14. Zerah L, Brochériou I, Galichon P, Peltier J, Hertig A. Warfarin- related nephropathy: a case report. Rev Med Interne. 2015;36:51-4.

15. Ware K, Brodsky P, Satoskar AA, Nadasdy T, Nadasdy G, Wu H, Rovin BH, Bhatt U, Von Visger J, Hebert LA, Brodsky SV. Warfarin-related nephropathy modelled by nephron reduction and excessive anticoagulation. J Am Soc Nephrol. 2011;22:1856-62.

16. Ozcan A, Ware K, Calomeni E, Nadasdy T, Forbes R, Satoskar AA, Nadasdy G, Rovin BH, Hebert LA, Brodsky SV. 5/6 nephrectomy as a validated rat model mimicking human warfarinrelated nephropathy. Am J Nephrol. 2012;35:356-64.

17. Ryan M, Ware K, Qamri Z, Satoskar A, Wu H, Nadasdy G, Rovin B, Hebert L, Nadasdy T, Brodsky SV. Warfarin-related nephropathy is the tip of the iceberg: direct thrombin inhibitor dabigatran induces glomerular hemorrhage with acute kidney injury in rats. Nephrol Dial Transpl. 2014;29:2228-34.

18. Escoli R, Santos P, Andrade S, Carvalho F. Dabigatran-related nephropathy in a patient with undiagnosed IgA nephropathy. Case Rep Nephrol. 2015;2015:298261. https ://doi. org/10.1155/2015/298261.

19. Stangier J, Feuring M. Using the HEMOCLOT direct thrombin inhibitor assay to determine plasma concentrations of dabigatran. Blood Coagul Fibrinolysis. 2012;23:138-43.

20. Samoš M, Stančiaková L, Ivanková J, Staško J, Kovář F, Dobrotová M, Galajda P, Kubisz P, Mokáň M. Monitoring of dabigatran therapy using Hemoclot (®) Thrombin Inhibitor assay in patients with atrial fibrillation. J Thromb Thrombolysis. 2015;39:95-100.

21. Heidbuchel H, Verhamme P, Alings M, Antz M, Hacke W, Oldgren J, Sinnaeve P, Camm AJ, Kirchhof P, Association European Heart Rhythm. European Heart Rhythm Association Practical Guide on the use of new oral anticoagulants in patients with nonvalvular atrial fibrillation. Europace. 2013;15:625-51.

22. De Caterina R, Camm AJ. What is 'valvular' atrial fibrillation? A reappraisal. Eur Heart J. 2014;35:3328-35.

23. Lip GY, Halperin JL. Improving stroke risk stratification in atrial fibrillation. Am J Med. 
2010;123:484-8.

24. Lip GY, Frison L, Halperin JL, Lane DA. Comparative validation of a novel risk score for predicting bleeding risk in anticoagulated patients with atrial fibrillation: the HAS-BLED (Hypertension, Abnormal Renal/Liver Function, Stroke, Bleeding History or Predisposition, Labile INR, Elderly, Drugs/Alcohol Concomitantly) score. J Am Coll Cardiol. 2011;57:173-80. 25. Clopper CJ, Pearson ES. The use of confidence or fiducial limits illustrated in the case of the binomial. Biometrika. 1934;26:404-13.

26. Liang KY, Zeger S. Longitudinal data analysis using generalized linear models. Biometrika. $1986 ; 73: 13-22$.

27. Brodsky SV, Mhaskar NS, Thiruveedi S, Dhingra R, Reuben SC, Calomeni E, Ivanov I, Satoskar A, Hemminger J, Nadasdy G, Hebert L, Rovin B, Nadasdy T. Acute kidney injury aggravated by treatment initiation with apixaban: another twist of anticoagulantrelated nephropathy. Kidney Res Clin Pract. 2017;36:387-92.

28. Connolly SJ, Ezekowitz MD, Yusuf S, Eikelboom J, Oldgren J, Parekh A, Pogue J, Reilly PA, Themeles E, Varrone J, Wang S, Alings M, Xavier D, Zhu J, Diaz R, Lewis BS, Darius H, Diener HC, Joyner CD, Wallentin L, RE-LY Steering Committee and Investigators. Dabigatran versus warfarin in patients with atrial fibrillation. N Engl J Med. 2009;361:1139-51.

29. Böhm M, Ezekowitz MD, Connolly SJ, Eikelboom JW, Hohnloser SH, Reilly PA, Schumacher H, Brueckmann M, Schirmer SH, Kratz MT, Yusuf S, Diener HC, Hijazi Z, Wallentin L. Changes in renal function in patients with atrial fibrillation: an analysis from the RE-LY trial. J Am Coll Cardiol. 2015;65:2481-93.

30. Chan YH, Yeh YH, See LC, Wang CL, Chang SH, Lee HF, Wu LS, Tu HT, Kuo CT. Acute kidney injury in asians with atrial fibrillation treated with dabigatran or warfarin. J Am Coll Cardiol. 2016;68:2272-83.

31. Yao X, Tangri N, Gersh BJ, Sangaralingham LR, Shah ND, Nath KA, Noseworthy PA. Renal outcomes in anticoagulated patients with atrial fibrillation. J Am Coll Cardiol. 2017;70:2621-32.

32. Eikelboom JW, Wallentin L, Connolly SJ, Ezekowitz M, Healey JS, Oldgren J, Yang S, Alings M, Kaatz S, Hohnloser SH, Diener HC, Franzosi MG, Huber K, Reilly P, Varrone J, Yusuf S. Risk of bleeding with 2 doses of dabigatran compared with warfarin in older and younger patients with atrial fibrillation: an analysis of the randomized evaluation of long-term 
anti-coagulant therapy (RE-LY) trial. Circulation. 2011;123:2363-72.

33. Owada S, Tomita H, Kinjo T, Ishida Y, Itoh T, Sasaki K, Horiuchi D, Kimura M, Sasaki S, Okumura K. CHA2DS2-VASc and HAS-BLED scores and activated partial thromboplastin time for prediction of high plasma concentration of dabigatran at trough. Thromb Res. 2015;135:627.

34. Pradaxa. Dabigatran etexilate: summary of the European public assessment report. https ://www.ema.europ a.eu/en/medic ines/ human /EPAR/prada xa. Accessed 24 Jan 2019. 
Table 1 Baseline characteristics of the patients. For quantitative variables the median values (first quartile, third quartile) are reported

\begin{tabular}{|c|c|c|c|c|}
\hline $\begin{array}{c}\text { Patient } \\
\text { characteristics }\end{array}$ & Total & DE110 & DE150 & $P$ value \\
\hline Female sex (\%) & $49.53 \%$ & 59.02 & 36.96 & 0.032 \\
\hline Age, y & $79(72.5-84)$ & $83(80-88)$ & 73 (69-77.5) & $<0.001$ \\
\hline BMI (kg/m2) & $26.17(23.23-28.42)$ & $26.76(23.81-28.66)$ & $25.71(22.04-28.30)$ & 0.213 \\
\hline CHA2DS2-VASc score & $5(3.5-5)$ & $5(4-6)$ & $4(3-5)$ & 0.001 \\
\hline HAS-BLED score & $3(2-3)$ & $3(2-3)$ & $2(2-3)$ & 0.119 \\
\hline Serum creatinine at TO & $0.93(0.78-1.06)$ & $0.96(0.76-1.08)$ & $0.88(0.79-1.00)$ & 0.323 \\
\hline eGFR at $\mathrm{T} \mathrm{ml} / \mathrm{min}$ & $63.01(49.56-76.05)$ & $56.40(41.25-69.55)$ & $67.75(57.57-77.74)$ & 0.002 \\
\hline DPC at T0 ng/ml & $102.9(70.2-164.6)$ & $106.30(71.25-171.85)$ & $99.00(63.15-149.97)$ & 0.389 \\
\hline DPC at T1, ng/ml & $115.65(80.12-166.97)$ & $128.90(82.25-175.05)$ & $99.2(76.0-124.70)$ & 0.072 \\
\hline
\end{tabular}

Table 2 Baseline characteristics of patients, according to DPC at T0

\begin{tabular}{|c|c|c|c|}
\hline $\begin{array}{c}\text { Patient } \\
\text { characteristics }\end{array}$ & $\mathrm{DPC}<200, \mathrm{ng} / \mathrm{ml}$ & $\mathrm{DPC} \geq 200, \mathrm{ng} / \mathrm{ml}$ & $P$ value \\
\hline Female sex (\%) & $42(46.67 \%)$ & $10(66.67 \%)$ & 0.174 \\
\hline Age, year & $80(74-86)$ & $84(81-87)$ & 0.136 \\
\hline BMI, kg/m2 & $26.2(23.29-28.65)$ & $25.84(21.37-27.84)$ & 0.558 \\
\hline CHA2DS2-VASc score & $4(4.00-5.75)$ & $5(5-6)$ & 0.015 \\
\hline HAS-BLED score & $3(2-3)$ & $2(2-3)$ & 0.250 \\
\hline Serum creatinine at TSTART , mg/dl, & $0.92(0.77-1.04)$ & $0.98(0.83-1.02)$ & 0.486 \\
\hline Serum creatinine at $\mathrm{T} 0, \mathrm{mg} / \mathrm{dl}$ & $0.90(0.76-1.02)$ & $1.00(0.82-1.29)$ & 0.053 \\
\hline eGFR at $\mathrm{TO}, \mathrm{ml} / \mathrm{min}$ & $65.98(53.56-77.58)$ & $47.23(36.49-61.53)$ & 0.001 \\
\hline
\end{tabular}


Table 3 Number and percentage of patients taking concomitant drugs

\begin{tabular}{l|rrrr}
\hline \multicolumn{1}{c|}{$\begin{array}{c}\text { Concomitant } \\
\text { drugs }\end{array}$} & \multicolumn{1}{c}{ Total } & DPC $<$ 200, ng/ml & DPC $\geq 200, \mathbf{n g} / \mathbf{m l}$ & P value \\
\hline ACE-inhibitors & $37(34.58 \%)$ & $29(32.22 \%)$ & $8(53.33 \%)$ & 0.146 \\
ARBs & $40(37.38 \%)$ & $36(40 \%)$ & $3(20 \%)$ & 0.161 \\
Diuretics & $62(57.94 \%)$ & $48(53.33 \%)$ & $12(80 \%)$ & 0.089 \\
Beta-blockers & $72(67.29 \%)$ & $58(64.44 \%)$ & $13(86.67 \%)$ & 0.135 \\
Proton pump inhibitors & $51(47.66 \%)$ & $45(50 \%)$ & $5(33.33 \%)$ & 0.274 \\
Verapamil & $1(0.93 \%)$ & $1(1.11 \%)$ & $0(0 \%)$ & 1.000 \\
Other calcium- & $26(24.3 \%)$ & $23(25.56 \%)$ & $3(20 \%)$ & 0.757 \\
antagonists & & & & 0.590 \\
Amiodarone & $7(6.54 \%)$ & $7(7.78 \%)$ & $2(13.33 \%)$ & 1.000 \\
Digoxin & $13(12.15 \%)$ & $11(12.22 \%)$ & $1(6.67 \%)$ & 0.143 \\
ASA & $2(1.87 \%)$ & $0(0 \%)$ & & \\
\hline
\end{tabular}


Figure 1

Study design: analytes dosed at each time point. TSTART time point before starting therapy with dabigatran etexilate, To time point 0 - study enrolment, $T_{1}$ time point $1-1$ week after enrolment, $T_{2}$ time point $2-2$ months after enrolment, $T_{3}$ time point $3-3$ months after enrolment, $S C r$ serum creatinine, $D P C$ dabigatran plasma concentration, $C y s C$ serum cystatin $\mathrm{C}$

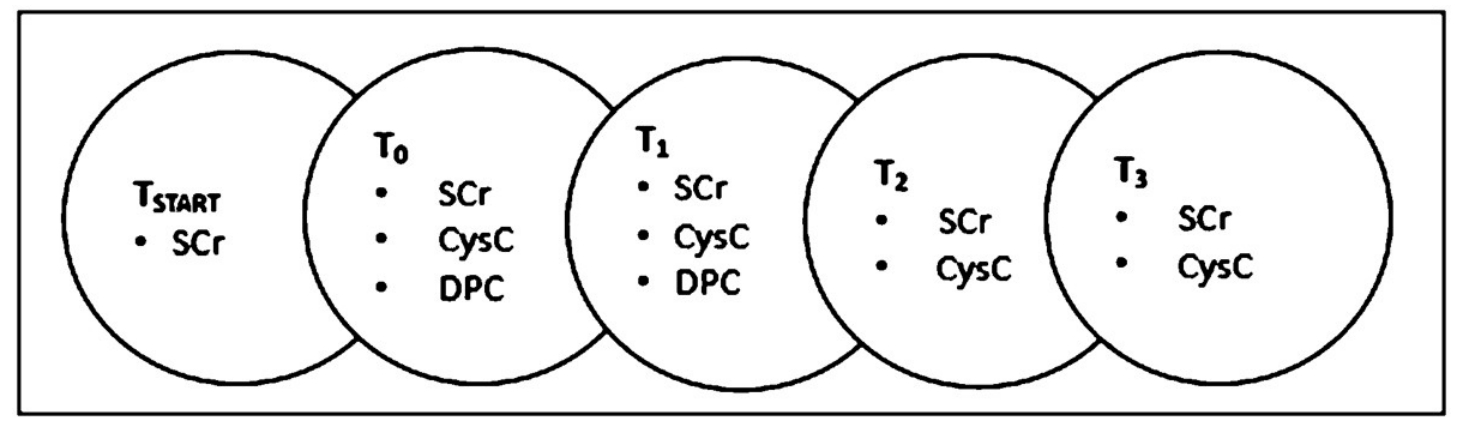

